\title{
One loop matching factors for staggered bilinear operators with improved glue
}

\author{
Jongjeong Kim*, Weonjong Lee \\ Frontier Physics Research Division and Center for Theoretical Physics \\ Department of Physics and Astronomy, Seoul National University, Seoul, 151-747, South Korea \\ E-mail: rvanguard@phya.snu.ac.kr, wlee@snu.ac.kr
}

\section{Stephen R. Sharpe}

Physics Department, University of Washington, Seattle, WA 98195-1560

E-mail: sharpe@phys.washington.edu

\begin{abstract}
We present results for matching factors for bilinear operators composed of HYP-smeared staggered fermions and constructed using HYP-smeared fat links. The matching factors are calculated perturbatively at one-loop order. The new feature of our calculation compared to previous work on HYP-smeared staggered fermions is the use of the Symanzik-improved gluon propagator, which allows our results to be applied to our ongoing simulations based on configurations generated by the MILC collaboration. We address the issue of the relative efficiency of various improvement schemes in reducing one-loop corrections to the matching factors.
\end{abstract}

The XXVII International Symposium on Lattice Field Theory - LAT2009 July 26-31 2009 Peking University, Beijing, China

\footnotetext{
* Speaker.
} 


\section{Introduction}

Matching factors are needed to convert matrix elements calculated on the lattice into physical observables in continuum renormalization schemes. We focus here on matching factors for the bilinear operators that we are using as part of an ongoing numerical study using MILC configurations. The valence fermions of which these operators are composed are HYP-smeared [1] improved staggered fermions, which thus differ from the sea quarks, which use the "asqtad" staggered action [2]. We calculate the matching factors (which are, in general, matrices) using perturbation theory at one-loop order. What is new compared to previous calculations using HYP-smeared staggered fermions $[3,4,5,6,7,8]$ is the use of the Symanzik-improved gluon action rather than the Wilson plaquette action. This generalization is necessary because the MILC configurations use an improved gluon action.

This work is part of a larger project, whose ultimate aim is to provide matching factors needed for the calculation of both quark masses [9] and $B_{K}[10,11,12,13]$. The results presented here allow us to study the relative impact of improving quark and gluon actions.

\section{Actions and Bilinear Operators}

Since sea-quarks enter first at the two-loop level (through vacuum polarization diagrams), we do not give the details of the sea-quark action.

For the valence quarks, we use the HYP-smeared staggered fermion action. This has the same form as the unimproved staggered fermion action,

$$
S_{f}=\frac{1}{2} \sum_{n, \mu} \eta_{\mu}(n) \bar{\chi}(n)\left(V_{\mu}(n) \chi(n+\hat{\mu})-V_{\mu}^{\dagger}(n-\hat{\mu}) \chi(n-\hat{\mu})\right)+m \sum_{n} \bar{\chi}(n) \chi(n),
$$

[with $n \in \mathbb{Z}^{4}$ a lattice coordinate and $\eta_{\mu}(n)=(-1)^{n_{1}+n_{2}+\cdots+n_{\mu-1}}$ ] but with the original thin links $U_{\mu}$ replaced with HYP-smeared links $V_{\mu}$. For the details of HYP-smearing, see Ref. [1]. This action has a number of important properties:

1. It substantially reduces the breaking of taste symmetry $[14,15,9]$;

2. It significantly reduces one loop corrections [7];

3. It performs tadpole improvement automatically [16];

4. It reduces scaling violations efficiently [17].

Given also its simplicity, it is an attractive choice for an improved staggered action.

The HYP-smeared links $V_{\mu}$ can be expressed in terms of blocked gauge fields $B_{\mu}$ :

$$
V_{\mu}(x)=\exp \left(i B_{\mu}(x+\hat{\mu} / 2)\right) .
$$

The blocked gauge fields $B_{\mu}$ can be written as a power series in the original gauge fields $A_{v}$,

$$
B_{\mu}=\sum_{n=1}^{\infty} B_{\mu}^{(n)}
$$


where $B_{\mu}^{(n)}$ contains all terms with $n$ powers of the gauge fields $A_{v}$. In the present one-loop calculation, we need only the linear term, $B_{\mu}^{(1)}$. One might have expected the quadratic term $B_{\mu}^{(2)}$ to enter as well, but its contribution vanishes due to the fact that HYP-smearing includes $S U(3)$ projections $[4,16]$.

The linear term can be written in terms of a blocking kernel $h_{\mu \nu}$, which it is convenient to define in momentum space:

$$
B_{\mu}^{(1)}(k)=\sum_{v} h_{\mu v}(k) A_{v}(k) .
$$

All information about the HYP-smearing, including the smearing parameters, is contained in $h_{\mu \nu}$. Following Ref. [7], we decompose the kernel into diagonal and off-diagonal parts

$$
h_{\mu v}(k)=\delta_{\mu v} D_{\mu}(k)+\left(1-\delta_{\mu v}\right) G_{\mu v}(k),
$$

With smearing coefficients chosen to remove $\mathscr{O}\left(a^{2}\right)$ taste symmetry breaking coupling at tree level ( $\alpha_{1}=0.75, \alpha_{2}=0.6$ and $\alpha_{3}=0.3$ in the notation of Ref. [1]), the diagonal part is

$$
D_{\mu}(k)=1-\sum_{v \neq \mu} \bar{s}_{v}^{2}+\sum_{\substack{v<\rho \\ v, \rho \neq \mu}} \bar{s}_{v}^{2} \bar{s}_{\rho}^{2}-\bar{s}_{v}^{2} \bar{s}_{\rho}^{2} \bar{s}_{\sigma}^{2},
$$

with $\bar{s}_{\mu}=\sin \left(k_{\mu} / 2\right)$, while the off-diagonal part is

$$
G_{\mu v}(k)=\bar{s}_{\mu} \bar{s}_{v}\left[1-\frac{\left(\bar{s}_{\rho}^{2}+\bar{s}_{\sigma}^{2}\right)}{2}+\frac{\bar{s}_{\rho}^{2} \bar{s}_{\sigma}^{2}}{3}\right] .
$$

For unimproved staggered fermions the blocking kernel simply reduces to $h_{\mu \nu}=\delta_{\mu \nu}$.

We now turn to the Symanzik-improved gluon action, which can be written as

$$
S_{g}=\frac{2}{g_{0}^{2}}\left[c_{\mathrm{pl}} \sum_{\mathrm{pl}} \operatorname{Re} \operatorname{Tr}\left(1-U_{\mathrm{pl}}\right)+c_{\mathrm{rt}} \sum_{\mathrm{rt}} \operatorname{Re} \operatorname{Tr}\left(1-U_{\mathrm{rt}}\right)+c_{\mathrm{pg}} \sum_{\mathrm{pg}} \operatorname{Re} \operatorname{Tr}\left(1-U_{\mathrm{pg}}\right)\right] .
$$

Here, $\mathrm{pl}, \mathrm{rt}$, and pg represent plaquette, rectangle and parallelogram, respectively. The coefficients $c_{i}$, where $i=\mathrm{pl}$, $\mathrm{rt}$, or $\mathrm{pg}$, should be chosen so as to improve the scaling behavior. In the present calculation, we use the tree-level improved coefficients

$$
c_{\mathrm{pl}}=\frac{5}{3}, \quad c_{\mathrm{rt}}=-\frac{1}{12}, \quad \text { and } \quad c_{\mathrm{pg}}=0,
$$

which were determined by Lüscher and Weisz $[18,19]$. The MILC collaboration actually use the 1-loop improved values for the coefficients, but this leads to changes which would enter only into a two-loop calculation. Note that the Wilson gauge action corresponds to $c_{\mathrm{pl}}=1, c_{\mathrm{rt}}=c_{\mathrm{pg}}=0$.

For the lattice bilinears, we use the operators of Ref. [20], which reside on $2^{4}$ hypercube. The operator with spin $S$ and taste $F$ can be written as

$$
[S \times F](y)=\frac{1}{16} \sum_{A, B}\left[\bar{\chi}_{b}(y+A)\left(\overline{\gamma_{S} \otimes \xi_{F}}\right)_{A B} \chi_{c}(y+B)\right] \mathscr{V}^{b c}(y+A, y+B),
$$

where $y$ is the coordinate of hypercube, and $A$ and $B$ denote the corners of hypercubes. Gauge invariance is maintained by the inclusion of $\mathscr{V}^{b c}(y+A, y+B)$, which is constructed by averaging over the shortest paths connecting $y+A$ and $y+B$, with each path constructed from the products of HYP-smeared links $V_{\mu}$. In this way the operators are improved in the same fashion as the action. This also ensures that the currents $[V \times S]$ and $[A \times P]$ are conserved. 


\section{Improved Gluon Propagator}

Propagators and vertices necessary to calculate perturbative corrections to the staggered bilinear operators can be found in Refs. $[3,4,5,6]$, with the exception of the gluon propagator for the Symanzik-improved action. Thus we discuss only the latter here.

The improved gluon propagator was worked out originally in Ref. [18]. We have found a convenient repackaging of the result, which we present here. This uses the notation ${ }^{1}$

$$
\hat{k}^{n} \equiv \sum_{\mu} \hat{k}_{\mu}^{n}, \quad \hat{k}_{\mu} \equiv 2 \sin \left(k_{\mu} / 2\right),
$$

and the following orthogonal projectors:

$$
\mathscr{P}_{\mu v}=\frac{\hat{k}_{\mu} \hat{k}_{v}}{\hat{k}^{2}}, \quad \delta_{\mu v}^{T}=\delta_{\mu v}-\mathscr{P}_{\mu v},
$$

The inverse gluon propagator with covariant gauge fixing can then be written

$$
\begin{aligned}
& \mathscr{D}_{\mu v}^{-1}=\frac{1}{\alpha} \hat{k}^{2} \mathscr{P}_{\mu v}+f \hat{k}^{2} \delta_{\mu v}^{T}-c \mathscr{M}_{\mu v}, \\
& \mathscr{M}_{\mu v}=\delta_{\mu v} \hat{k}_{\mu}^{2} \hat{k}^{2}-\hat{k}_{\mu}^{3} \hat{k}_{v}-\hat{k}_{\mu} \hat{k}_{v}^{3}+\frac{\hat{k}_{\mu} \hat{k}_{v} \hat{k}^{4}}{\hat{k}^{2}},
\end{aligned}
$$

where $\alpha$ is the gauge-fixing parameter,

$$
f=\left(\omega-c^{\prime} \hat{k}^{2}-c \hat{k}^{4} / \hat{k}^{2}\right),
$$

and

$$
\omega=c_{\mathrm{pl}}+8 c_{\mathrm{rt}}+8 c_{\mathrm{pg}}, \quad c=c_{\mathrm{rt}}-c_{\mathrm{pg}}, \quad c^{\prime}=c_{\mathrm{pg}} .
$$

Since we use gauge-invariant operators the matching factors are independent of $\alpha$ and we chose $\alpha=1$. For the tree-level improved Symanzik action $\omega=1, c=-1 / 12$ and $c^{\prime}=0$.

Inverting $\mathscr{D}_{\mu \nu}^{-1}$, we find the improved gluon propagator to be

$$
\mathscr{D}_{\mu v}=\alpha \frac{\mathscr{P}_{\mu v}}{\hat{k}^{2}}+\frac{\left[\hat{k}^{2}\left(\hat{k}^{2}-\tilde{c} x_{1}\right)+\tilde{c}^{2} x_{2}\right] \delta_{\mu v}^{T}+\tilde{c}\left(\hat{k}^{2}-\tilde{c} x_{1}\right) \mathscr{M}_{\mu v}+\tilde{c}^{2}\left(\mathscr{M}^{2}\right)_{\mu v}}{f\left\{\hat{k}^{2}\left[\hat{k}^{2}\left(\hat{k}^{2}-\tilde{c} x_{1}\right)+\tilde{c}^{2} x_{2}\right]-\tilde{c}^{3} x_{3}\right\}},
$$

where $\tilde{c}=c / f$, and

$$
\begin{aligned}
x_{1} & =\operatorname{Tr}(\mathscr{M})=\left(\hat{k}^{2}\right)^{2}-\hat{k}^{4}=2 \sum_{\mu<v} \hat{k}_{\mu}^{2} \hat{k}_{v}^{2}, \\
x_{2} & =\frac{1}{2}\left[\operatorname{Tr}^{2}(\mathscr{M})-\operatorname{Tr}\left(\mathscr{M}^{2}\right)\right]=\hat{k}^{2}\left[\hat{k}^{6}-(3 / 2) \hat{k}^{2} \hat{k}^{4}+(1 / 2)\left(\hat{k}^{2}\right)^{3}\right]=3 \hat{k}^{2} \sum_{\mu<v<\rho} \hat{k}_{\mu}^{2} \hat{k}_{v}^{2} \hat{k}_{\rho}^{2}, \\
x_{3} & =\frac{1}{6}\left[\operatorname{Tr}^{3}(\mathscr{M})-3 \operatorname{Tr}(\mathscr{M}) \operatorname{Tr}\left(\mathscr{M}^{2}\right)+2 \operatorname{Tr}\left(\mathscr{M}^{3}\right)\right] \\
& =\frac{\left(\hat{k}^{2}\right)^{2}}{6}\left[\left(\hat{k}^{2}\right)^{4}+3\left(\hat{k}^{4}\right)^{2}-6 \hat{k}^{4}\left(\hat{k}^{2}\right)^{2}+8 \hat{k}^{6} \hat{k}^{2}-6 \hat{k}^{8}\right]=4\left(\hat{k}^{2}\right)^{2} \hat{k}_{1}^{2} \hat{k}_{2}^{2} \hat{k}_{3}^{2} \hat{k}_{4}^{2} .
\end{aligned}
$$

\footnotetext{
${ }^{1}$ Be careful that $\hat{k}^{4} \neq\left(\hat{k}^{2}\right)^{2}$ in this notation.
} 


\section{Renormalization of Bilinear Operators}

The Feynman diagrams relevant to the one-loop renormalization of the bilinear operators are shown in Fig. 1. Analytic expressions for these diagrams using the unimproved gluon propagator can be found in Refs. [7, 8]. It turns out that the generalization needed when using the improved gluon propagator is relatively simple. One simply replaces the composite gluon propagator from a smeared link in the $\mu$ 'th direction to a smeared link in the $v^{\prime}$ th direction (which is a building block of the calculation) as follows:

$$
\left(1 / \hat{k}^{2}\right) \sum_{\lambda} h_{\mu \lambda} h_{v \lambda} \rightarrow \sum_{\alpha \beta} h_{\mu \alpha} h_{v \beta} \mathscr{D}_{\alpha \beta}
$$

The key simplification arises from the fact that this composite propagator contains off-diagonal terms even when the gluon propagator is diagonal (as it is for the Wilson gauge action in Feynman gauge), so that no new types of contribution arise when moving to the (off-diagonal) improved gluons propagator. One must simply evaluate the loop integrals (which is done numerically) using the more complicated composite gluon propagator.

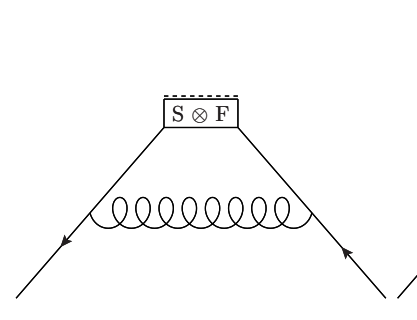

(a)

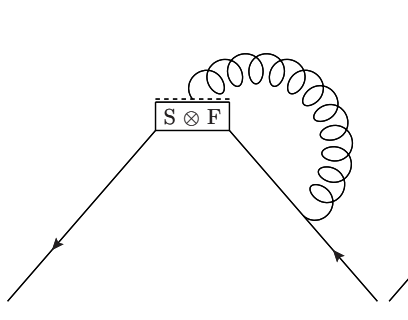

(b)

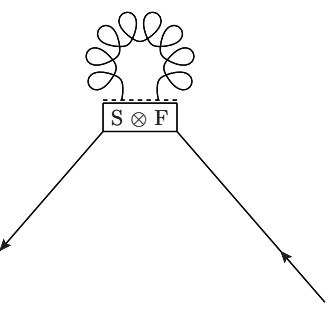

(c)

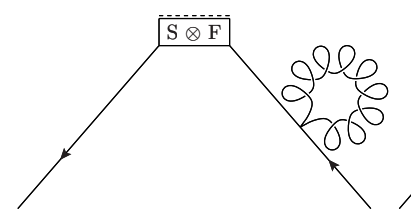

(d)

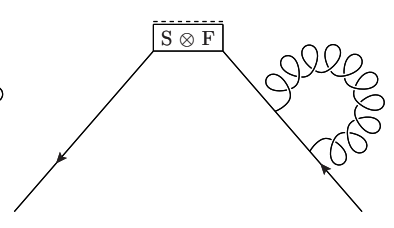

(e)

Figure 1: Feynman diagrams for bilinear operators

Two independent calculations have been done as a cross-check. In the end, we obtain the one-loop renormalization factors of the bilinear operators,

$$
O_{i}^{\mathrm{Latt},(1)}=\left\{\delta_{i j}+\frac{4}{3} \frac{g^{2}}{(4 \pi)^{2}}\left[\gamma_{i j} \log (a \lambda)+C_{i j}^{\mathrm{Latt}}\right]\right\} O_{j}^{\mathrm{Latt},(0)}+\mathscr{O}(a),
$$

where the superscripts indicate the order in perturbation theory, $i$ and $j$ are indices which run over the 256 bilinear operators, $\lambda$ is the "gluon mass" used to regularize the infrared divergences, and $\gamma_{i j}$ is the anomalous dimension matrix. The quantities of interest are $C_{i j}^{L a t t}$, the finite parts of the corrections. These have, in general, non-zero off-diagonal elements. A similar expression holds in continuum regularizations, and by combining with the result (4.2) one can obtain the desired one-loop matching factors. Full details will be presented in Ref. [21]. Here we simply quote, in Tables 1 and 2, results for some representative diagonal and off-diagonal elements of $C_{i j}^{L a t t}$. 
As noted in Ref. [7], one can apply the mean-field improvement method of Ref. [22] to our HYP-smeared operators. This should reduce the size of fluctuations in the gauge links. This results in shifts in the values of the diagonal elements $C_{i i}^{\text {Latt }}[4,5,6]$. Mean-field improved values of $C_{i i}^{\text {Latt }}$ are shown in the last four columns of Table 1.

\begin{tabular}{l|rrrr|rrrr}
\hline \hline Operator & $(a)$ & $(b)$ & \multicolumn{1}{c}{$(c)$} & \multicolumn{1}{c}{$(d)$} & $(a)^{\prime}$ & $(b)^{\prime}$ & $(c)^{\prime}$ & $(d)^{\prime}$ \\
\hline$(1 \otimes 1)$ & 41.73 & 2.60 & 32.82 & 1.91 & 31.86 & 1.54 & 25.59 & 1.18 \\
$\left(\gamma_{5} \otimes 1\right)$ & -35.86 & -7.17 & -27.24 & -6.01 & -6.25 & -4.01 & -5.55 & -3.84 \\
$\left(\gamma_{\mu} \otimes 1\right)$ & 0.00 & 0.00 & 0.00 & 0.00 & 0.00 & 0.00 & 0.00 & 0.00 \\
$\left(\gamma_{\mu} \otimes \xi_{5}\right)$ & -22.51 & -3.97 & -17.04 & -3.10 & -2.77 & -1.86 & -2.58 & -1.65 \\
$\left(\gamma_{\mu \nu} \otimes 1\right)$ & -10.97 & -1.84 & -8.38 & -1.34 & -1.10 & -0.79 & -1.15 & -0.62 \\
$\left(\gamma_{\mu \nu} \otimes \xi_{\rho \sigma}\right)$ & -34.05 & -5.19 & -25.64 & -3.82 & -4.44 & -2.03 & -3.95 & -1.65 \\
\hline \hline
\end{tabular}

Table 1: Diagonal part of representative diagonal coefficients $C_{i i}^{\text {Latt }}$. Note that $\mu, v, \rho$, and $\sigma$ are all different. Results are given for the following four choices of actions: $(a)$ thin links (in the fermion action and bilinears) with the Wilson plaquette action; (b) HYP-smeared links with the Wilson plaquette action; $(c)$ thin links with the improved gluon action; $(d)$ HYP-smeared links with the improved gluon action. The prime in the labels indicates that mean-field improvement is applied. Results are accurate to the number of digits quoted.

\begin{tabular}{lllrrrr}
\hline \hline Name & Operator- $i$ & Operator- $j$ & $(a)$ & $(b)$ & $(c)$ & $(d)$ \\
\hline$c_{V V M}$ & $\left(\gamma_{\mu} \otimes \xi_{v}\right)$ & $\left(\gamma_{\mu} \otimes \xi_{\mu}\right)$ & -3.042 & -0.351 & -2.495 & -0.321 \\
$c_{V A M}$ & $\left(\gamma_{\mu} \otimes \xi_{\mu 5}\right)$ & $\left(\gamma_{\mu} \otimes \xi_{v 5}\right)$ & 0.647 & 0.257 & 0.609 & 0.244 \\
$c_{V T M}$ & $\left(\gamma_{\mu} \otimes \xi_{\mu v 5}\right)$ & $\left(\gamma_{\mu} \otimes \xi_{\rho v 5}\right)$ & 1.486 & 0.280 & 1.292 & 0.266 \\
$c_{T A M}$ & $\left(\gamma_{\mu \nu} \otimes \xi_{\mu 5}\right)$ & $\left(\gamma_{\mu \nu} \otimes \xi_{\rho 5}\right)$ & 0.676 & -0.006 & 0.547 & -0.003 \\
\hline \hline
\end{tabular}

Table 2: Off-diagonal coefficients $C_{i j}^{l a t}$. The notation is the same as in Table 1 . Results are accurate to the number of digits quoted.

\section{Discussion}

We can use the results to compare the reduction in the size of one-loop matching factors achieved by different improvement schemes. For vector currents and the off-diagonal coefficients, the anomalous dimensions vanish and the $C_{i j}^{\text {lat }}$ give a direct measure of the size of the corrections. Looking at the results for $\left(\gamma_{\mu} \otimes \xi_{5}\right)$, a vector current containing 3 links, one sees that the corrections in column (a) for unimproved fermions and glue are reduced by all choices of improvement scheme. Comparing the impact of applying each improvement alone, the greatest reduction is achieved by mean-field improvement, with HYP-smearing following close behind, but with the improved gauge action alone leading to a much smaller reduction. Combining all three improvements leads to the smallest coefficients. Similar results holds for the off-diagonal coefficients of Table 2, except that these are unaffected by mean-field improvement.

For the scalar and tensor operators, which have non-zero anomalous dimensions, the coefficients $C_{i j}^{l a t}$ depend on the choice of infrared regularization. Thus one should consider the difference 
between the coefficients for fixed spin and differing tastes, i.e. the differences between the first and second rows and between the fifth and sixth rows of Table 1. For these differences one finds a similar pattern of improvements to those noted above, except that for the scalar bilinears HYP smearing leads to a significantly greater reduction than mean-field improvement.

Overall, we conclude that improving the gluon action reduces the one-loop corrections somewhat but is not nearly as effective in this regard as HYP-smearing.

\section{Acknowledgments}

The research of J. Kim and W. Lee is supported by the Creative Research Initiatives program (3348-20090015) of the KOSEF grant funded by the Korean government (MEST). The work of S. Sharpe is supported in part by the US DOE grant no. DE-FG02-96ER40956.

\section{References}

[1] A. Hasenfratz and F. Knechtli, Phys. Rev. D 64, 034504 (2001) [arXiv:hep-lat/0103029].

[2] C. Bernard and et al., Phys. Rev. D 64, 054506 (2001) [arXiv:hep-lat/0104002].

[3] D. Daniel and S. Sheard, Nucl. Phys. B 302, 471 (1988).

[4] A. Patel and S. R. Sharpe, Nucl. Phys. B 395, 701 (1993) [arXiv:hep-lat/9210039].

[5] N. Ishizuka and Y. Shizawa, Phys. Rev. D 49, 3519 (1994) [arXiv:hep-lat/9308008].

[6] W. Lee and M. Klomfass, Phys. Rev. D 51, 6426 (1995) [arXiv:hep-lat/9412039].

[7] W. Lee and S. R. Sharpe, Phys. Rev. D 66, 114501 (2002) [arXiv:hep-lat/0208018].

[8] W. Lee and S. R. Sharpe, Phys. Rev. D 68, 054510 (2003) [arXiv:hep-lat/0306016].

[9] T. Bae et al., Phys. Rev. D 77, 094508 (2008) [arXiv:0801.3000 [hep-lat]].

[10] Taegil Bae, et al., PoS LAT2009, 261 (2009) [arXiv:xxxx.xxxx [hep-lat]].

[11] Hyung-Jin Kim, et al., PoS LAT2009, 262 (2009) [arXiv:Xxxx.xxxx [hep-lat]].

[12] Boram Yoon, et al., PoS LAT2009, 263 (2009) [arXiv:xxxx.xxxx [hep-lat]].

[13] Jangho Kim, et al., PoS LAT2009, 264 (2009) [arXiv:xxxx.xxxx [hep-lat]].

[14] T. Bae, J. Kim, W. Lee and S. R. Sharpe, PoS LAT2006, 166 (2006) [arXiv:hep-lat/0610056].

[15] T. Bae et al., PoS LAT2007, 089 (2007) [arXiv:0710.0017 [hep-lat]].

[16] Weonjong Lee, Phys. Rev. D 66, 114504 (2002) [arXiv:hep-lat/0208032].

[17] Weonjong Lee, PoS LAT2006, 015 (2006) [arXiv:hep-lat/0610058].

[18] P. Weisz, Nucl. Phys. B 212, 1 (1983).

[19] M. Luscher and P. Weisz, Commun. Math. Phys. 97, 59 (1985) [Erratum-ibid. 98, 433 (1985)].

[20] H. Kluberg-Stern et al., Nucl. Phys. B 220, 447 (1983).

[21] J. Kim, W. Lee, and S. R. Sharpe, in preparation.

[22] G. P. Lepage and P. B. Mackenzie, Phys. Rev. D 48, 2250 (1993) [arXiv:hep-lat/9209022]. 